\title{
Genome-wide association studies in ARDS: SNPing the tangled web of heterogeneity
}

\author{
Sara Clohisey ${ }^{1}$ and Pratik Sinha $2^{2^{*}}$
}

๑ 2021 Springer-Verlag GmbH Germany, part of Springer Nature

Genome-wide association studies (GWAS) have demonstrated the power of genomics in helping us better understand complex diseases. A GWAS involves sequencing hundreds-of-thousands to millions of DNA variants (single nucleotide polymorphisms; SNPs) in individual patients who display a phenotype or trait. Comparison of cases to appropriately selected controls reveals statistically significant DNA differences potentially responsible for the observed phenotype. Once specific variants or genes have been implicated as a risk-modifier, the next challenges are to link them to functional biological pathways that may in turn lead to identification of therapies. As an illustration, in Type II Diabetes Mellitus, GWAS and gene-resequencing studies have identified a protective loss-of-function gene mutation that encodes a zinc transporter in pancreatic islet cells that in turn has led to an interest in developing therapies that inhibit this zinc-transporter [1]. Similar GWAS-led successes have also been seen for repurposing or identifying therapies in autoimmune diseases [2].

In heterogeneous clinical syndromes such as ARDS, identification of novel biomarkers, such as genetic variants, represent a tantalizing strategy for stratifying patients and identifying effective treatments [3]. By virtue of its non-specific definition, however, the likelihood of identifying such biomarkers in ARDS is lower than in more specifically defined diseases. Prior GWAS studies have sought to identify genetic risk factors for ARDS [4-6]. The diversity of the studied populations, aetiology, and pathophysiology in ARDS has most likely contributed to the observed discordant findings between these

\footnotetext{
*Correspondence: p.sinha@wustl.edu

2 Department of Anesthesiology, Washington University Medical School, 660 S. Euclid Ave, Campus, Box 8054, St. Louis, MO 63110, USA

Full author information is available at the end of the article
}

studies. A convincing GWAS would require thousands of samples, however, could these separate, smaller GWAS studies be combined to uncover new answers?

In this issue of the journal, Du et al. set out to connect data from these past studies, and identify novel genetic loci associated with ARDS [7]. Their innovative approach makes use of seemingly disparate data to produce novel results through the creative and robust application of an array of computational biology approaches. The authors first integrated data from individuals of European ancestry, the iSPAAR and MESSI studies, identifying a novel genetic variant rs7967111. Next, they combined data sets of African American ancestry from the MESSI study and one further study [3] and identified a second novel locus, rs619652. Importantly, these findings point to a genetic architecture that differs between European and African American ancestries, mandating future studies to focus on this discrepancy to ensure more equitable healthcare innovation. The authors perform a trans-ethnic metaanalysis using the available data sets to identify potential genetic factors relating to all-cause ARDS in individuals across multiple ancestries. Interestingly, no common genetic variants were found to be significant when the European and African American ancestry groups are analysed together. However, gene set enrichment analysis, which looks for over-represented proteins or pathways in a data set, found shared cellular processes. While genetic variation may not be common between the two ancestries, the pathways affected might well be, highlighting the role of genetic pleiotropy in complex disease states.

The authors leveraged both in-house and publically available data to perform functional analyses of rs7967111 and identified BORCS5 and DUSP16 as downstream genes whose expression in blood is potentially dysregulated through this SNP. Again, using publicly available data repositories, they found these genes were 


\section{A Current ARDS}

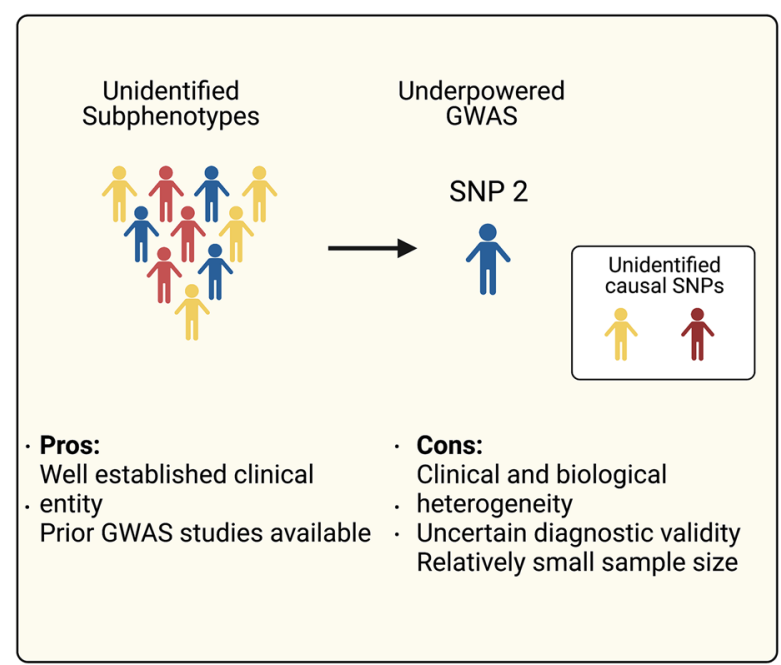

\section{Future ARDS}

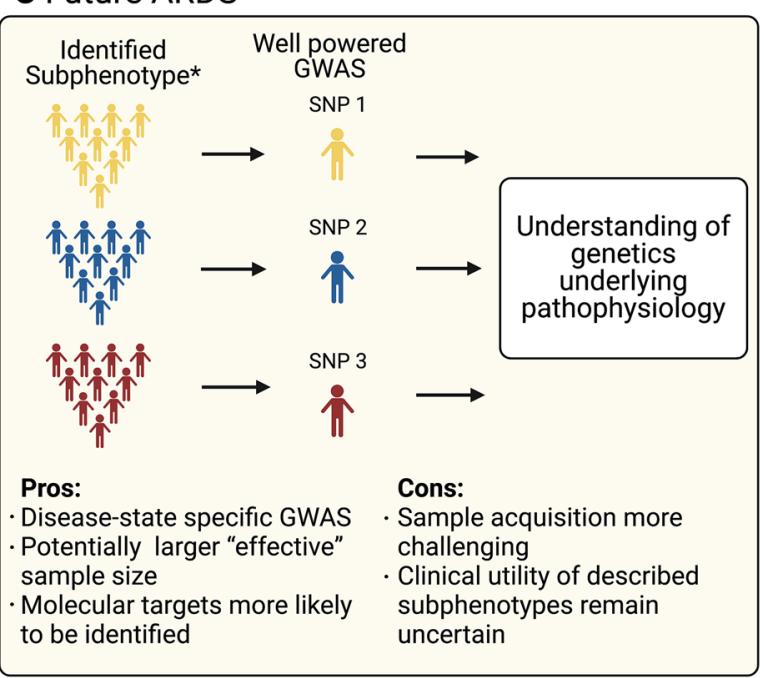

\section{B Current COVID-19}

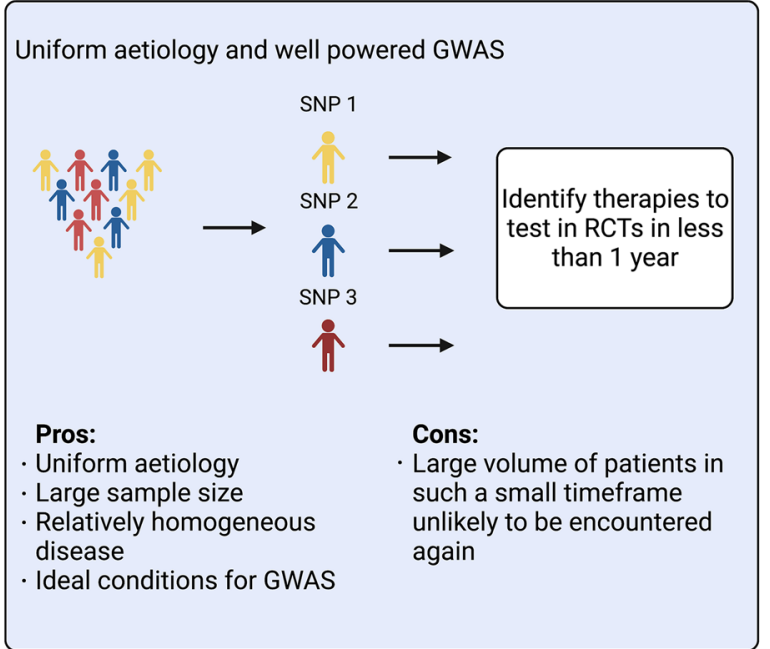

\section{Future Critical Care}

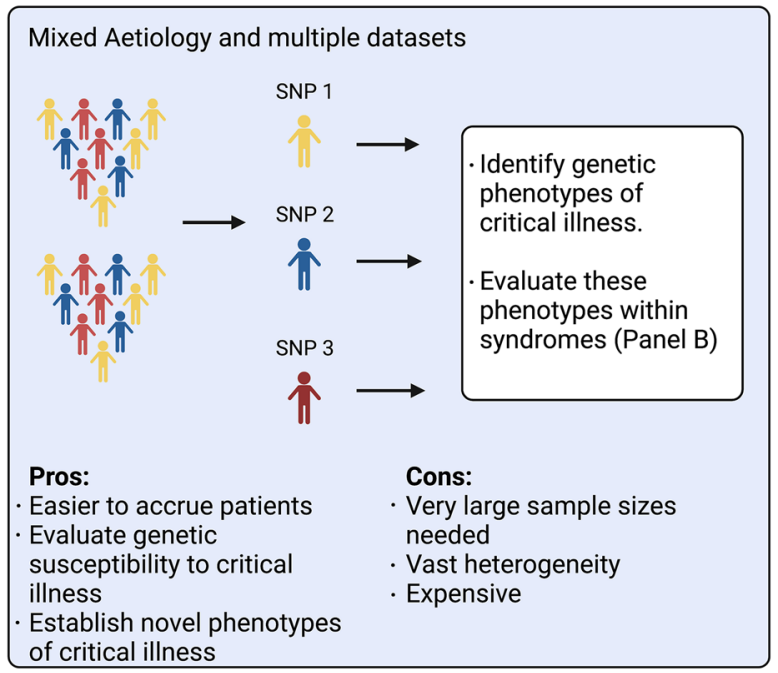

Fig. 1 Overview of frameworks for GWAS studies in critical illness. A Current approach to ARDS GWAS. B Current approach to COVID-19 GWAS (close to ideal). C Proposed approach to ARDS GWAS (disease-state specific); *Subphenotypes could be related to aetiology or based on disease states, e.g., the hyperinflammatory phenotype. D Proposed approach to Critical Care GWAS (large multicentre studies embracing heterogeneity and overlap of ARDS and sepsis). Image created using BioRender (www.biorender.com)

prominently expressed among lung and immune cells in humans and in mice treated with lipopolysaccharide, giving face validity to their findings.

The authors acknowledge many of the limitations of their studies including the limited sample size, specifically, in the African-American cohort. They also acknowledge the ethnic diversity of African-American individuals. Nevertheless, this is, to our knowledge, the largest GWAS in African-American patients with ARDS and is commendable despite the limited sample size.
Another limitation, albeit a thought-provoking one, are the analyses that seeks to compare the findings in all-cause ARDS to COVID-19. An inherent assumption of this comparison is that, genetically, ARDS development is uniform regardless of aetiology. We know, however, biologically not all ARDS are the same [3]. Putting aside the diagnostic unreliability of the Berlin definition of ARDS, a simple question for the field to ask is-do we expect genetic-susceptibility for patients meeting the Berlin definition either due to bacterial pneumonia, trauma, aspiration, sepsis or COVID-19, to be uniform? 
It may be that there is some loose commonality in the end manifestation of lung injury, such as hypoxemic respiratory failure, beyond that, however, the biological diversity due to these disparate insults makes this idea seem improbable. Importantly, in the unlikely scenario that a novel molecular target may be identified using genomebased data, without a robust diagnostic marker for ARDS translating this to prospective treatment still represents a considerable challenge.

Challenges of studying biology in clinical syndromes such as ARDS and sepsis are not insurmountable but require developing strong networks of research infrastructure. GWAS in all-cause ARDS, to date, have universally been under-powered. One possibility may be to perform GWAS in established biological or aetiological subphenotypes of ARDS [3]. Though the overall sample size may be smaller, by studying genomics of biologically uniform subgroups, the effective sample size may, paradoxically, be enhanced. Alternatively, given the overlap of sepsis and ARDS, an intuitive way forward may be to study common genetic variants that lead to critical illness, irrespective of the nominal clinical placeholders with which we label our patients (Fig. 1). In either case, concerted efforts to gather biospecimens as part of routine clinical workflow would be needed to fully realise the potential of GWAS in critical illness, and as such, represents an urgent research agenda for the specialty.

The current COVID-19 pandemic serves as a timely illustration of the advantages of studying a uniform aetiology, namely SARS-CoV-2. In addition, the sheer volume of COVID-19 has allowed sample collection among in-patients at an unprecedented level. Together, these factors have contributed to the rapidity with which GWAS studies have been translated to potential molecular targets, as illustrated by the Severe COVID GWAS group [8] and GenOMICC [9] studies. The latter study identified four novel loci, and using Mendelian Randomisation implicated IFNAR2 and TYK2, both members of the JAK/STAT signalling pathway, as potential therapeutic targets. Baricitinib, a JAK/STAT inhibitor that authorised for treating autoimmune disorders such as rheumatoid arthritis [10] and atopic dermatitis [11], has shown some efficacy in reducing recovery time in patients with COVID-19 [12] and is now included in RECOVERY, a national clinical trial across UK hospitals investigating potential treatments for COVID-19 [13]. An unknown, but surely substantial, proportion of the vast number of patients included in various COVID-19 GWAS will have presented with ARDS, but the relevance of this to non-COVID-19 ARDS remains uncertain. It is likely that findings from these COVID-19 studies could inform future ICU GWAS studies.
Despite the numerous challenges of GWAS in ARDS, in their study, Du and colleagues have continued to lead and push the field forward and provided an innovative investigative pipeline translating SNPs to therapies. From a bigger picture stand-point, as more biologically sophisticated approaches to understanding disease emerge, critical care must decide whether it continues to cling to broad clinical definitions for such studies or revert back to a more biologically intuitive framework for classifying patients.

\section{Author details \\ ${ }^{1}$ The Roslin Institute, Edinburgh EH25 9RG, Scotland, UK. ${ }^{2}$ Department of Anesthesiology, Washington University Medical School, 660 S. Euclid Ave, Campus, Box 8054, St. Louis, MO 63110, USA. \\ Declarations \\ Conflicts of interest \\ On behalf of all authors, the corresponding author states that there is no conflict of interest.}

\section{Publisher's Note}

Springer Nature remains neutral with regard to jurisdictional claims in published maps and institutional affiliations.

Received: 4 May 2021 Accepted: 16 June 2021

Published online: 26 June 2021

\section{References}

1. Flannick J, Thorleifsson G, Beer NL, Jacobs SBR, Grarup N, Burtt NP et al (2014) Loss-of-function mutations in SLC30A8 protect against type 2 diabetes. Nat Genet 46:357-363. https://doi.org/10.1038/ng.2915

2. Visscher PM, Wray NR, Zhang Q, Sklar P, McCarthy MI, Brown MA et al (2017) 10 years of GWAS discovery: biology, function, and translation. Am J Hum Genet 101:5-22. https://doi.org/10.1016/.ajhg.2017.06.005

3. Wilson JG, Calfee CS (2020) ARDS subphenotypes: understanding a heterogeneous syndrome. Crit Care. https://doi.org/10.1186/ s13054-020-2778-x

4. Bime C, Pouladi N, Sammani S, Batai K, Casanova N, Zhou T et al (2018) Genome-wide association study in African Americans with acute respiratory distress syndrome identifies the selectin P ligand gene as a risk factor. Am J Respir Crit Care Med 197:1421-1432. https://doi.org/10.1164/ rccm.201705-09610C

5. Reilly JP, Wang F, Jones TK, Palakshappa JA, Anderson BJ, Shashaty MGS et al (2018) Plasma angiopoietin-2 as a potential causal marker in sepsisassociated ARDS development: evidence from Mendelian randomization and mediation analysis. Intensive Care Med 44:1849-1858. https://doi. org/10.1007/s00134-018-5328-0

6. Guillen-Guio B, Lorenzo-Salazar JM, Ma SF, Hou PC, Hernandez-Beeftink T, Corrales A et al (2020) Sepsis-associated acute respiratory distress syndrome in individuals of European ancestry: a genome-wide association study. Lancet Respir Med 8:258-266. https://doi.org/10.1016/S22132600(19)30368-6

7. Du M, Garcia JGN, Christie JD et al (2021) Integrative omics provide biological and clinical insights into acute respiratory distress syndrome. Intensive Care Med. https://doi.org/10.1007/s00134-021-06410-5

8. Severe Covid-19 GWAS Group, Ellinghaus D, Degenhardt F, Bujanda L, But M, Albillos A et al (2020) Genomewide association study of severe COVID19 with respiratory failure. N Engl J Med 383:1522-1534. https://doi.org/ 10.1056/NEJMoa2020283 
9. Pairo-Castineira E, Clohisey S, Klaric L, Bretherick AD, Rawlik K, Pasko D et al (2021) Genetic mechanisms of critical illness in COVID-19. Nature 591:92-98. https://doi.org/10.1038/s41586-020-03065-y

10. Genovese MC, Smolen JS, Takeuchi T, Burmester G, Brinker D, Rooney TP et al (2020) Safety profile of baricitinib for the treatment of rheumatoid arthritis over a median of 3 years of treatment: an updated integrated safety analysis. Lancet Rheumatol 2:e347-e357. https://doi.org/10.1016/ S2665-9913(20)30032-1

11. Guttman-Yassky E, Silverberg Jl, Nemoto O, Forman SB, Wilke A, Prescilla $R$ et al (2019) Baricitinib in adult patients with moderate-to-severe atopic dermatitis: a phase 2 parallel, double-blinded, randomized placebo-controlled multiple-dose study. J Am Acad Dermatol 80:913-921.e9. https:// doi.org/10.1016/j.jaad.2018.01.018

12. Kalil AC, Patterson TF, Mehta AK, Tomashek KM, Wolfe CR, Ghazaryan V et al (2021) Baricitinib plus Remdesivir for hospitalized adults with COVID19. N Engl J Med. 384:795-807. https://doi.org/10.1056/NEJMoa2031994

13. ISRCTN50189673 (2020) [cited 2021 Jun 24] A randomised trial of treatments to prevent death in patients hospitalised with COVID-19 (coronavirus). https://doi.org/10.1186/ISRCTN50189673 